\title{
MiR-195-5p Reduces Esophageal Cancer Cell Proliferation Through The IGF-1R/AKT Axis
}

Zhao-xian Lin ( $\square$ haley033@163.com )

Fujian Provincial Hospital

Xing Lin

Fujian Provincial Hospital

Lihuan Zhu

Fujian Provincial Hospital

Jian-yuan Huang

Fujian Provincial Hospital

Yang-yun Huang

Fujian Provincial Hospital

\section{Research Article}

Keywords: Esophageal cancer, GLUT1, IGF-1R, AKT, miR-195-5p.

Posted Date: June 16th, 2021

DOI: https://doi.org/10.21203/rs.3.rs-569371/v1

License: (c) (i) This work is licensed under a Creative Commons Attribution 4.0 International License. Read Full License 


\section{Abstract \\ Background}

Esophageal cancer (ECa) remains a major cause of mortality across the globe. The expression of MiR$195-5 p$ is altered in a plethora of tumors, but its role in ECa development and progression are undefined.

\section{Result}

Here, we show that miR-195-5p is downregulated in ECa and associated with poor survival in ECa. Function assays indicated that MiR-195-5p inhibited ECa progression. Mechanistically, we identified IGF$1 \mathrm{R}$ as a downstream target of miR-195-5p, and miR-195-5p/IGFR axis caused a loss of GLUT1 expression, reduced glucose uptake, reduced lactate production, and low levels of ATP production.

\section{Conclusion}

Collectively, miR-195-5p as a Eca suppressor impaired glycolysis. This highlighting miR-195-5p as a novel target for much needed anti-ECa therapeutics.

\section{Introduction}

Compared with other regions, the incidence of esophageal cancer in Chinese men is particularly high and poses a global threat to human health [1]. Advances in diagnosis and treatment have improved ECa treatment, the efficiency of ECa treatment and subsequent patient prognosis are still poor[2]. Therefore, ECA remains a global health burden and urgently needs new diagnoses and effective treatment strategies [3]. It is necessary to further understand the molecular regulators of ECa tumorigenesis.

MicroRNAs (miRNAs) are non-coding RNAs that range in size from 20-24 nucleotides. MiRNAs regulate gene expression in all mammalian cell-types [4-6]. The dysregulation of miRNAs leads to a plethora of human pathologies, including carcinogenesis and tumorigenesis, amongst other disease states [7-9]. Notable examples include miR-154 in ECa cells and tissues [10] and miR-139-3p that is suppressed in glioblastoma due to its ability to slow cell growth and metastasis [11]. Emerging evidence links other miRNAs in ECa[12,13]. Tang and colleagues revealed that miR-204-5p suppresses ECa growth through its effects on IL-11[14], whilst Li and coworkers highlighted the tumor suppressor functions of miR-671-5p through its ability to suppress FGF2 expression and activity [15]. MiR-195 is a known tumor-suppressor in lung cancer [16], prostate cancer[17]and cervical cancer[18]. However, the mechanism(s) mediating the involvement of miR-195-5p in ECa development remains undefined.

Aerobic glycolysis occurs in an array of cancer cell types and endows tumor cells with the ability to process glucose through glycolytic enzymes in the absence of oxygen produced from mitochondrial respiration $[19,20]$. To maintain rapid cancer cell growth, glucose transporters (GLUTs) and glycolytic 
enzyme activity are required, and are frequently over-expressed in tumor cells. IGF-1R phosphorylates and activates AKT as a result of insulin stimulation, leading to the up-regulation of GLUTs and their transport to the cell-surface [21-23]. MiRNAs targeting the IGF-1R-AKT-GLUT1 signaling axis are promising anticancer agents. Here, we assessed the effects of miR-195-5p on tumor cell proliferation and invasion via the IGF-1R-AKT axis in ECa cells, in an attempt to identify this signaling axis as a novel target for much needed-ECa therapeutics.

\section{Materials And Methods}

\section{Tissue specimens}

A total of 121 pairs of ECa tumor and normal para-cancerous tissues were obtained from patients undergoing surgery at the Fujian Provincial Hospital. Enrolled patients had not undergone preoperative systemic or local anti-tumor therapy. Following collection, pathological examination was used to confirm ECa diagnosis, and samples were snap frozen prior to storage at $-80^{\circ} \mathrm{C}$. All patients supplied informed consent to participate, and the Ethics Committee of the Fujian Provincial Hospital approved all human studies, the Ethics number was NO. FJPH-2013.06.05. All experiments were performed in accordance with the relevant guidelines and regulations.

\section{Cell culture}

Human endothelial HET-1A cells and ECa cells including EC9706 and TE-1 were grown in DMEM $+10 \%$ FBS at $37^{\circ} \mathrm{C}$. ECa ECA109 and TE-8 cells were grown in RPMI- $1640+10 \% \mathrm{FBS}$ at $37^{\circ} \mathrm{C}$. HEK293 cells were grown in MEM $+10 \%$ FBS at $37^{\circ} \mathrm{C}$. For overexpression studies, TE-1 cells were transfected with miR-195$5 p$ mimics or miR-195-5p negative controls (NC; $40 \mathrm{nM}$ ) for $24 \mathrm{~h}$. For suppression studies, TE-8 cells were transfected with miR-574-3p inhibitors or miR-195-5p inhibitor NCs.

\section{Reporter assays}

Potential miR-195-5p targets were identified using the TargetScan database, which highlighted IGF-1R as a potential target. Based on this prediction, pmiR-RB-report plasmids (RiboBio Inc) were cloned to contain either a mutant or wild-type version of the 3'-UTR of IGF-1R. Plasmids were then co-transfected with appropriate miRNA mimics, inhibitors, or controls for $48 \mathrm{~h}$. Commercial kits (Promega) were used to analyze luciferase activity. Firefly activity was normalized to Renilla.

\section{Western blotting}

Cell lines or tissues were lysed in RIPA (Beyotime) buffer and protein content of the lysates was assessed via BCA assays (Thermo Fisher). Proteins ( $20 \mathrm{ng}$ ) were resolved by SDS-PAGE electrophoresis and transferred. PVDF membranes were blocked in $5 \%$ milk and probed overnight with antibodies against IGF1R (1:1000, 9750S, CST), p-AKT (Ser473, 1:1000, 9271S; CST), and total-AKT (1:1000, 9272S, CST), GLUT1 (1:1000, sc-377228; Santa Cruz) at $4^{\circ} \mathrm{C}$. Blots were then labeled for $1 \mathrm{~h}$ with anti-mouse or anti- 
rabbit HRP-secondary antibodies. Proteins were visualized using the ECL system on a ChemiDoc Imaging Platform (Bio-Rad).

\section{Real-time PCR}

RNA was isolated from cell lines or paraffin-embedded tissue using a miRNeasy FFPE kits (Qiagen) or miRNeasy Mini kits (Qiagen). cDNA was generated using a miScript II RT kit (Qiagen) from $1 \mu \mathrm{g}$ of input RNA or miRNA. RT-PCR parameters were as follows: $37^{\circ} \mathrm{C}$ for $60 \mathrm{~min}, 95^{\circ} \mathrm{C}$ for $5 \mathrm{~min}$. The miScript SYBR Green PCR kit was used for qRT-PCR. Reactions were performed as follows: $95^{\circ} \mathrm{C}$ for $15 \mathrm{~min} ; 40$ cycles of $94^{\circ} \mathrm{C}$ for $15 \mathrm{~s}, 55^{\circ} \mathrm{C}$ for $30 \mathrm{~s}$, and $70^{\circ} \mathrm{C}$ for $30 \mathrm{~s}$ on a 7500 Fast Real-Time PCR platform. Values were normalized to GAPDH and $\mathrm{U} 6$ and quantified. All reactions were performed in triplicate.

MiR-195-5p Forward 5'-ACACTCCAGCTGGGTGTCAGTTTGTCAAAT-3';

MiR-195-5p Reverse 5'-CTCAACTGGTGTCGTGGAGTCGGCAATTCAGT-3',

U6 Forward 5'-TGCGGGTGCTCGCTTCGCAGC-3';

U6 Reverse 5'-CCAGTGCAGGGTCCGAGGT-3',

IGF-1R Forward5'-GAGGTGGGCTCGGGAGAAGAT-3';

IGF-1R Reverse5'-TTCACCACACCCTTGGGCAAC-3',

GAPDH Forward 5'-TTGGTATCGTGGAAGGACTCA-3';

GAPDH Reverse 5'-TGTCATCATATTTGGCAGGTT-3'.

\section{Cell Proliferation Assays}

CCK-8 assays were used to assess ECa and non-ECa cell proliferation. Cells were co-transfected as described in $96-$ well plates (1,000 cells/well) for $24 \mathrm{~h}$, and $10 \mu \mathrm{l}$ of CCK-8 was added to the wells in normal culture conditions for $3 \mathrm{~h}$. Absorbances at $450 \mathrm{~nm}$ were read on a Bio-Rad microplate reader.

\section{Colony Formation Assays}

Treated cells $\left(1 \times 10^{3}\right.$ per well) were seeded into $10 \mathrm{~cm}$ culture dishes for 2 weeks. Cell colonies were stained in crystal violet (1\%) and colony numbers were counted.

\section{Transwell assay}

For migration/invasion assays, 12-well plates containing Transwell inserts (Corning, MA, USA) with $8 \mu \mathrm{m}$ pore sizes were used. For migration assays, $\sim 100,000$ cells were resuspended in serum-free media and added to the upper regions of the Transwell chambers for $8 \mathrm{~h}$ at $37^{\circ} \mathrm{C}$. Cells in the lower chambers were then removed and methanol fixed prior to staining using $0.04 \%$ crystal violet. Cells were then quantified. 
For invasion assays, the protocols were identical to those above, but the transwell inserts had first been coated using Matrigel (BD Biosciences, MA, USA), and plates were instead incubated for $24 \mathrm{~h}$.

\section{Glucose uptake, lactate, and ATP assays}

Colorimetric assays were performed to assay glucose uptake. Briefly, cells ( $1 \times 10^{4}$ per 96 wells) were glucose starved in Krebs-solution plus 2\% BSA for $40 \mathrm{~min}$. Cells were then treated with 2-DG (10 mM) for $20 \mathrm{~min}$ and color changes were assessed on a plate reader. For the assessment of lactate and ATP production, cells $\left(5 \times 10^{5}\right)$ were homogenized in commercial assay buffers at $4^{\circ} \mathrm{C}$. Soluble fractions were assayed using Lactate Assay Kit II reagent or ATP colometric assays.

\section{Statistical analysis}

Data are shown and the mean \pm SD analyzed via SPSS 17.0. Inter-group differences were assessed via a student's t-test. Multiple groups were compared using a one-way ANOVA analysis of variance. Kaplan Meier (KM) curves were plotted for survival analysis, and groups were compared via log-rank assessments ( $\mathrm{n}=3$ for all). P-values $<0.05$ were deemed significant differences

\section{Results}

\section{MiR-195-5p is suppressed and correlated with poor survival in $\mathrm{ECa}$}

We first investigated miR-195-5p levels in ECa vs. non-cancer cells and tissues via qRT-PCR analysis. MiR195-5p expression was found to be lower in ECa tissues compared to healthy tissues (Fig. 1A) with 96/121(79.34\%) of ECa samples showing lower levels of the miRNA (Fig. 1B). In terms of cancer stage, miR-195-5p levels were lower in T3 + T4 tumor depths compared to T1 + T2 tissues (Fig. 1C), and in TNM3 + 4 stage samples compared to TNM1 + 2 (Fig. 1D). MiR-195-5p levels were also lower in samples showing + ve lymph node metastasis compared to non-metastatic samples (Fig. 1E). RT-PCR analysis further showed that miR-195-5p was downregulated in ECa cell lines vs. non-ECa HECC cells. Amongst the cell-types, miR-195-5p showed the lowest levels of expression in TE-1 cells, and the highest levels of expression in TE-8 cells (Fig. 1F). These data confirmed that miR-195-5p expression is suppressed in ECa tissues and cells. Following miR-195-5p expression were divided into high- and low-group according to the median levels measured in ECa tissues. Table 1 shows that low levels of miRNA-195-5p positively correlated with several clinical features such as advanced TNM staging $(P=0.004)$, invasion depth $(P=$ 0.008 ) and lymph node metastasis $(P<0.001)$. KM curves suggested that low levels miR-195-5p were associated with poor survival (Fig. $1 \mathrm{G}$ and $\mathrm{H}$ ). These data confirmed that miRNA-195-5p was suppressed and correlated with poor survival in ECa. 
Table 1

The correlation between clinicopathological parameters and miR-195-5p expression in human esophageal cancer

\begin{tabular}{|c|c|c|c|c|}
\hline \multirow[t]{3}{*}{ Clinical features } & \multirow[t]{3}{*}{ Total } & \multicolumn{2}{|c|}{ miR-195-5p } & \multirow[t]{3}{*}{$p$-vaule } \\
\hline & & High & Low & \\
\hline & & $(N=61)$ & $(N=60)$ & \\
\hline Age (years) & & & & 0.210 \\
\hline$<60$ & 36 & 15 & 21 & \\
\hline$\geq 60$ & 85 & 46 & 39 & \\
\hline Gender & & & & 0.792 \\
\hline Male & 68 & 35 & 33 & \\
\hline Female & 53 & 26 & 27 & \\
\hline Differentiation grade & & & & 0.521 \\
\hline Well & 41 & 19 & 22 & \\
\hline Moderate + Poor & 80 & 42 & 38 & \\
\hline TNM stage & & & & 0.004 \\
\hline$|+| \mid$ & 76 & 46 & 30 & \\
\hline III & 45 & 15 & 30 & \\
\hline Depth of invasion & & & & 0.008 \\
\hline $\mathrm{T} 1+\mathrm{T} 2$ & 57 & 36 & 21 & \\
\hline $\mathrm{T} 3+\mathrm{T} 4$ & 64 & 25 & 39 & \\
\hline Lymph node metastasis & & & & $<0.001$ \\
\hline No & 80 & 53 & 27 & \\
\hline Yes & 41 & 8 & 33 & \\
\hline
\end{tabular}

MiR-195-5p inhibits the metastatic phenotypes of ECa cells in vitro

We next explored the mechanistic effects of miR-195-5p in ECa cells. RT-PCR analysis revealed that miR195-5p expression increased in TE-1 cells transfected with miRNA mimics, whilst miR-195-5p levels were lower in TE-8 cells expressing miR-195-5p inhibitors (Fig. 2A). CCK-8 assays showed that the miR-195-5p mimics reduced TE-1 cell proliferation, whilst miR-195-5p inhibitors increased TE-8 cell growth (Fig. 2B-C). MiR-195-5p mimics also led to a loss of colony counts, whilst miR-195-5p silencing increased colony 
numbers (Fig. 2D). We further investigated the effects of miR-195-5p on ECa cell migration and invasion in Transwell assays. TE-1 migration was suppressed following miR-195-5p overexpression, whilst the migration and invasiveness of TE-8 cells increased in the presence of miR-195-5p inhibitors (Fig. 2E-F).

\section{Mir-195-5p Suppresses Igf-1r Expression In Eca}

To further investigate the regulatory mechanisms of miR-195-5p in ECa cells, TargetScan analysis was performed to predict cellular targets, identifying IGF-1R as a novel candidate (Fig. 3A). Dual-luciferase reporter assays showed that the transfection of miR-195-5p mimics led to a loss of luciferase activity in cell expressing IGF1R-WT reporters, but no differences were observed in cells expressing mutant IGF1R (Fig. 3B). IGF1R expression was also suppressed by miR-195-5p mimics in TE-1 cells, but increased following miR-195-5p silencing in TE-8 cells (Fig. 3C-D). GEPIA (gene expression profile Ingenuity analysis) data further indicated that IGF-1R is overexpressed in ECa tissues compared to non-ECa tissues (Fold change $>1.5, \mathrm{P}<0.05$, Fig. $3 \mathrm{E}$ ) which was experimentally confirmed the mRNA level via RT-PCR analysis (Fig. 3F). These data revealed the negative correlation between miR-195-5p and IGF-1R in ECa tissue (Fig. 3G), highlighting the miR-195-5p/IGF-1R axis as a novel therapeutic strategy in ECa.

\section{MiR-195-5p regulates glycolysis in ECa cells via the IGF-1R/AKT/GLUT1 pathway}

IGF-1R activates AKT and GLUT1 expression, inducing cancer cell glycolysis. We thus investigated AKT signaling and GLUT1 expression in ECa cells. We found that miR-195-5p overexpression led to a loss of $p$ AKT (S473) and GLUT1 expression in TE-1 cells, whilst miR-195-5p silencing enhanced their levels in TE-8 cells (Fig. 4A). This revealed miR-195-5p is a negative regulator of the IGF-1R-AKT-GLUT1 axis in ECa cells. As GLUT1 regulates glucose uptake, we next investigated the effects of miR-195-5p on ECa cell glycolysis. We found that miR-195-5p mimics causes a decrease in TE-1 cellular glucose uptake (Fig. 4B), subsequently leading to a loss of lactate in ECa cells relative to control cells (Fig. 4C). The metabolic processing of glucose leads to ATP production, and as such, we investigated the effects of miR-195-5p on ATP levels. We found that the miRNA mimics decreased ATP levels in TE-1 cells (Fig. 4D), consistent with that observed for glucose uptake and lactate production. In contrast, in cells expressing miR-195-5p inhibitors, the depletion of miR-195-5p increased glucose uptake and lactate production (Fig. 4B-C). Similarly, miR-195-5p inhibitors enhanced ATP production (Fig. 4D). Collectively, these data highlight miR$195-5 p$ as a negative regulator of glycolysis in ECa cells.

\section{MiR-195-5p reduces ECa cell proliferation, invasion and glycolysis through IGF-1R}

We next examined whether miR-195-5p influences glycolysis through its targeting and suppression of IGF-1R in ECa cells. To achieve this, we overexpressed IGF-1R in TE-1 miR-195-5p mimic cell lines, or silenced IGF-1R in TE-8 miR-195-5p inhibitor cell lines (Fig. 5A and Figure S1). CCK-8 assays showed that miR-195-5p mimics inhibited TE-1 cell proliferation, whilst IGF-1R overexpression attenuated these effects. Moreover, miR-195-5p inhibitors led to enhanced TE-8 cell proliferation, which was alleviated through IGF-1R silencing (Fig. 5B). Transwell assays showed that the loss of ECa cell migration and 
invasion induced by miR-195-5p mimics increased following IGF-1R overexpression. The enhanced levels of cell migration and invasion induced by miR-195-5p inhibitors also decreased in IGF-1R silenced cells (Fig. 5C-D). Furthermore, as shown in Fig. 5E-G, miR-195-5p mimics led to a loss of glucose uptake, decreased lactate production and impaired ATP generation, all of which were reversed by IGF-1R overexpression in miR-195-5p-transfected cells. MiR-195-5p inhibitors also led to enhanced glucose uptake, lactate production and ATP generation; effects that were alleviated through IGF-1R silencing (Fig. 5E-G). These data collectively highlight the role of aerobic glycolysis in the regulation of the proliferation and invasion of ECa cells via miR-195-5p.

\section{Discussion}

In this study, lower levels of miR-195-5p expression were observed in ECa tumors and cells, which was associated with advantaged TNM staging $(P=0.004)$, invasion depth $(P=0.008)$, metastasis $(P<0.001)$, and poor survival. Moreover, miR-195-5p inhibited the proliferation and metastatic phenotypes of ECa cells through its ability to target IGF-1R and inhibit its expression, the loss of IGF-1R activity led to a concomitant decrease in pAKT and GLUT1 expression. The loss of miR-195-5p also led to a loss of glucose uptake, lactate production, and ATP production through the IGF-1R/AKT/GLUT1 axis. These findings collectively highlight miR-195-5p is a key regulator of ECa tumorigenesis.

Glycolysis is enhanced in tumor vs. healthy tissue, due to metabolic reprogramming[24, 25]. This endows tumor cells with energy reserves that promote cell proliferation[26]. Glycolysis can be targeted to inhibit tumor progression. MiRNAs target components of glycolysis including GLUT1, G6PD, and LDHA[27-30] providing a mechanism of cancer cell targeting[31]. We show that miR-195-5p inhibits glycolysis and suppresses ECa cell proliferation through IGF-1R. This reveals new roles for this important miRNA in both cancer cell proliferation and survival.

IGF-1R is upregulated in an array of human cancers, including HCC, renal cell carcinoma and gastric cancer[32-34]. IGF-1R silencing leads to a 50\% decrease in the size of mouse embryos[35]. IGF-1R interacts with IGF- 1 to regulate tumorigenesis, proliferation and metastasis through PI3K/AKT and MAPK/ERK[36, 37]. IGF-1R also activates PI3K/AKT to regulate cancer cell proliferation and invasiveness[38-41]. Conversely the inhibition of IGF-1R functionality represents a therapeutic avenue for anti-cancer strategies. Indeed, drugs targeting IGF-1R are currently in clinical trials for a multitude of human cancers[42].

AKT or protein kinase B is activated through phosphorylation on its hydrophobic motif (Ser473) and activation loop (Thr308) in response to growth factors including IGF[43]. P-AKT is the active form of AKT that is known to phosphorylate and regulate an array of cell activities[44]. Zhang and colleagues showed that AKT is upregulated in cancer and associated with tumor aggressiveness and poor prognosis in HCC patients[45]. In addition, the overexpression of pAKT is associated with a poor prognosis many important human carcinomas[46-49]. The inhibition of AKT is a known and well-characterized target for cancer therapy. Studies have reported that PI3K/AKT signaling regulates GLUT1 trafficking and activity[50, 51]. 
PI3K/AKT inhibition leads to a loss of GLUT1 expression, and decreased glucose metabolism, suppressing the proliferation of colorectal cancer cells[52]. In this study, we found that the overexpression of miR-195-5p decreased IGF-1R expression in ECa cells, thereby suppressing AKT phosphorylation and GLUT1 expression. This led to a loss of glucose uptake, decreased lactate production, and reduced ATP levels in the presence of high levels of miR-195-5p, highlighting miR-195-5p as a novel negative regulator of glycolysis.

Collectively, these data highlight miR-195-5p as a tumor suppressor that is downregulated in ECa cells to impair glycolysis. This highlights miR-195-5p as a novel target for much needed anti-ECa therapeutic interventions. These findings now warrant further investigation in in vivo models of ECa.

\section{Declarations}

\section{Ethics approval and consent to participate}

All patients supplied informed consent to participate, and the Ethics Committee of the Fujian Provincial Hospital approved all human studies, the Ethics number was NO. FJPH-2013.06.05.

\section{Consent for publication}

N/A

\section{Availability of data and materials}

The datasets used and/or analysed during the current study available from the corresponding author on reasonable request.

\section{Competing interests}

None to declare.

\section{Funding}

$\mathrm{N} / \mathrm{A}$

\section{Authors' contributions}

Zhao-xian Lin and Li-huan Zhu wrote the main manuscript text and All authors prepared figures and tables. All authors reviewed the manuscript.

\section{Acknowledgements}

$\mathrm{N} / \mathrm{A}$

\section{References}


1. Torre LA, Bray F, Siegel RL, Ferlay J, Lortet-Tieulent J, Jemal A. Global cancer statistics, 2012. CA: a cancer journal for clinicians. 2015; 65: 87-108.

2. Short MW, Burgers KG, Fry VT. Esophageal Cancer. American family physician. 2017; 95: 22-8.

3. Chen W, Zheng R, Baade PD, Zhang S, Zeng H, Bray F, et al. Cancer statistics in China, 2015. CA: a cancer journal for clinicians. 2016; 66: 115-32.

4. Li J, Liang Y, Lv H, Meng H, Xiong G, Guan X, et al. miR-26a and miR-26b inhibit esophageal squamous cancer cell proliferation through suppression of c-MYC pathway. Gene. 2017; 625: 1-9.

5. Huang Y, Shen XJ, Zou Q, Wang SP, Tang SM, Zhang GZ. Biological functions of microRNAs: a review. Journal of physiology and biochemistry. 2011; 67: 129-39.

6. Hu Y, Liu Q, Zhang M, Yan Y, Yu H, Ge L. MicroRNA-362-3p attenuates motor deficit following spinal cord injury via targeting paired box gene 2. Journal of integrative neuroscience. 2019; 18: 57-64.

7. Lujambio A, Lowe SW. The microcosmos of cancer. Nature. 2012; 482: 347-55.

8. Iorio MV, Ferracin M, Liu CG, Veronese A, Spizzo R, Sabbioni S, et al. MicroRNA gene expression deregulation in human breast cancer. Cancer research. 2005; 65: 7065-70.

9. Wu WK, Lee CW, Cho CH, Fan D, Wu K, Yu J, et al. MicroRNA dysregulation in gastric cancer: a new player enters the game. Oncogene. 2010; 29: 5761-71.

10. Wang J, Fang Y, Liu YF, Wang X, Wang XL, Wang RY, et al. MiR-154 inhibits cells proliferation and metastasis in melanoma by targeting AURKA and serves as a novel prognostic indicator. European review for medical and pharmacological sciences. 2019; 23: 4275-84.

11. Shi L, Yuan Y, Li HY. MicroRNA-139-3p suppresses growth and metastasis of glioblastoma via inhibition of NIN1/RPNI2 binding protein 1 homolog. European review for medical and pharmacological sciences. 2019; 23: 4264-74.

12. Feber A, Xi L, Luketich JD, Pennathur A, Landreneau RJ, Wu M, et al. MicroRNA expression profiles of esophageal cancer. The Journal of thoracic and cardiovascular surgery. 2008; 135: 255 - 60; discussion 260.

13. He B, Yin B, Wang B, Xia Z, Chen C, Tang J. MicroRNAs in esophageal cancer (review). Molecular medicine reports. 2012; 6: 459-65.

14. Tang J, AUID- Oho, Li Z, Zhu Q, Wen W, Wang J, et al. miR-204-5p regulates cell proliferation, invasion, and apoptosis by targeting IL-11 in esophageal squamous cell carcinoma. Journal of cellular physiology. 2020; 235: 3043-55.

15. Li X, Nie C, Tian B, Tan X, Han W, Wang J, et al. miR-671-5p Blocks The Progression Of Human Esophageal Squamous Cell Carcinoma By Suppressing FGFR2. International journal of biological sciences. 2019; 15: 1892-904.

16. Liu B, Qu J, Xu F, Guo Y, Wang Y, Yu H, et al. MiR-195 suppresses non-small cell lung cancer by targeting CHEK1. Oncotarget. 2015; 6: 9445-56.

17. Cai C, Chen QB, Han ZD, Zhang YQ, He HC, Chen JH, et al. miR-195 Inhibits Tumor Progression by Targeting RPS6KB1 in Human Prostate Cancer. Clinical cancer research: an official journal of the 
American Association for Cancer Research. 2015; 21: 4922-34.

18. Shen CJ, Cheng YM, Wang CL. LncRNA PVT1 epigenetically silences miR-195 and modulates EMT and chemoresistance in cervical cancer cells. Journal of drug targeting. 2017; 25: 637-44.

19. Akram M. Mini-review on glycolysis and cancer. Journal of cancer education: the official journal of the American Association for Cancer Education. 2013; 28: 454-7.

20. Zheng J. Energy metabolism of cancer: Glycolysis versus oxidative phosphorylation (Review). Oncology letters. 2012; 4: 1151-7.

21. Lenz G, Hamilton A, Geng S, Hong T, Kalkum M, Momand J, et al. t-Darpp Activates IGF-1R Signaling to Regulate Glucose Metabolism in Trastuzumab-Resistant Breast Cancer Cells. Clinical cancer research: an official journal of the American Association for Cancer Research. 2018; 24: 1216-26.

22. Wang Y, Zhao X, Shi D, Chen P, Yu Y, Yang L, et al. Overexpression of SIRT1 promotes high glucoseattenuated corneal epithelial wound healing via p53 regulation of the IGFBP3/IGF-1R/AKT pathway. Investigative ophthalmology \& visual science. 2013; 54: 3806-14.

23. Garg N, Thakur S, McMahan CA, Adamo ML. High fat diet induced insulin resistance and glucose intolerance are gender-specific in IGF-1R heterozygous mice. Biochemical and biophysical research communications. 2011; 413: 476-80.

24. Osada H, Takahashi T. MicroRNAs in biological processes and carcinogenesis. Carcinogenesis. 2007; 28: $2-12$.

25. Svoronos AA, Engelman DM, Slack FJ. OncomiR or Tumor Suppressor? The Duplicity of MicroRNAs in Cancer. Cancer research. 2016; 76: 3666-70.

26. Li XB, Gu JD, Zhou QH. Review of aerobic glycolysis and its key enzymes - new targets for lung cancer therapy. Thoracic cancer. 2015; 6: 17-24.

27. Fan JY, Yang Y, Xie JY, Lu YL, Shi K, Huang YQ. MicroRNA-144 mediates metabolic shift in ovarian cancer cells by directly targeting Glut1. Tumour biology: the journal of the International Society for Oncodevelopmental Biology and Medicine. 2016; 37: 6855-60.

28. Liu M, Gao J, Huang Q, Jin Y, Wei Z. Downregulating microRNA-144 mediates a metabolic shift in lung cancer cells by regulating GLUT1 expression. Oncology letters. 2016; 11: 3772-6.

29. Li P, Yang X, Cheng Y, Zhang X, Yang C, Deng X, et al. MicroRNA-218 Increases the Sensitivity of Bladder Cancer to Cisplatin by Targeting Glut1. Cellular physiology and biochemistry: international journal of experimental cellular physiology, biochemistry, and pharmacology. 2017; 41: 921-32.

30. Zhao X, Lu C, Chu W, Zhang B, Zhen Q, Wang R, et al. MicroRNA-124 suppresses proliferation and glycolysis in non-small cell lung cancer cells by targeting AKT-GLUT1/HKII. Tumour biology: the journal of the International Society for Oncodevelopmental Biology and Medicine. 2017; 39 : 1010428317706215.

31. Hamanaka RB, Chandel NS. Targeting glucose metabolism for cancer therapy. The Journal of experimental medicine. 2012; 209: 211-5. 
32. E C, Li J, Shao D, Zhang D, Pan Y, Chen L, et al. The insulin-like growth factor-I receptor inhibitor picropodophyllin-induced selective apoptosis of hepatocellular carcinoma cell through a caspasedependent mitochondrial pathway. Oncology research. 2013; 21: 103-10.

33. Sichani MM, Yazdi FS, Moghaddam NA, Chehrei A, Kabiri M, Naeimi A, et al. Prognostic value of insulin- like growth factor-I receptor expression in renal cell carcinoma. Saudi journal of kidney diseases and transplantation: an official publication of the Saudi Center for Organ Transplantation, Saudi Arabia. 2010; 21: 69-74.

34. Gryko M, Kisluk J, Cepowicz D, Zinczuk J, Kamocki Z, Guzinska-Ustymowicz K, et al. Expression of insulin-like growth factor receptor type 1 correlate with lymphatic metastases in human gastric cancer. Polish journal of pathology: official journal of the Polish Society of Pathologists. 2014; 65: $135-40$.

35. Efstratiadis A. Genetics of mouse growth. The International journal of developmental biology. 1998; 42: 955-76.

36. Cao Z, Liu LZ, Dixon DA, Zheng JZ, Chandran B, Jiang BH. Insulin-like growth factor-I induces cyclooxygenase-2 expression via PI3K, MAPK and PKC signaling pathways in human ovarian cancer cells. Cellular signalling. 2007; 19: 1542-53.

37. Guo T, Feng Y, Liu Q, Yang X, Jiang T, Chen Y, et al. MicroRNA-320a suppresses in GBM patients and modulates glioma cell functions by targeting IGF-1R. Tumour biology: the journal of the International Society for Oncodevelopmental Biology and Medicine. 2014; 35: 11269-75.

38. Pollak MN. Insulin-like growth factors and neoplasia. Novartis Foundation symposium. 2004; 262: 84-98; discussion 98-107, 265-8.

39. Werner $\mathrm{H}$, LeRoith $\mathrm{D}$. The role of the insulin-like growth factor system in human cancer. Advances in cancer research. 1996; 68: 183-223.

40. Pollak M. The insulin and insulin-like growth factor receptor family in neoplasia: an update. Nature reviews. Cancer. 2012; 12: 159-69.

41. King H, Aleksic T, Haluska P, Macaulay VM. Can we unlock the potential of IGF-1R inhibition in cancer therapy. Cancer treatment reviews. 2014; 40: 1096-105.

42. Hartog H, Wesseling J, Boezen HM, van der Graaf WT. The insulin-like growth factor 1 receptor in cancer: old focus, new future. European journal of cancer: official journal for European Organization for Research and Treatment of Cancer (EORTC) [and] European Association for Cancer Research (EACR). 2007; 43: 1895-904.

43. Wang L, Guo W, Ma J, Dai W, Liu L, Guo S, et al. Aberrant SIRT6 expression contributes to melanoma growth: Role of the autophagy paradox and IGF-AKT signaling. Autophagy. 2018; 14: 518-33.

44. Vivanco I, Sawyers CL. The phosphatidylinositol 3-Kinase AKT pathway in human cancer. Nature reviews. Cancer. 2002; 2: 489-501.

45. Zhang Y, Guo X, Yang M, Yu L, Li Z, Lin N. Identification of AKT kinases as unfavorable prognostic factors for hepatocellular carcinoma by a combination of expression profile, interaction network analysis and clinical validation. Molecular bioSystems. 2014; 10: 215-22. 
46. Chen YL, Chen PM, Ming YZ, Lin PY, Chu CP, Chu PY. Phosphorylated AKT expression in tumoradjacent normal tissue is associated with poor prognosis in patients with hepatocellular carcinoma. Oncology letters. 2017; 14: 7461-6.

47. Malinowsky K, Nitsche U, Janssen KP, Bader FG, Spath C, Drecoll E, et al. Activation of the PI3K/AKT pathway correlates with prognosis in stage II colon cancer. British journal of cancer. 2014; 110: 2081-9.

48. Tokunaga E, Kimura Y, Oki E, Ueda N, Futatsugi M, Mashino K, et al. Akt is frequently activated in HER2/neu-positive breast cancers and associated with poor prognosis among hormone-treated patients. International journal of cancer. Journal international du cancer. 2006; 118: 284-9.

49. Hager M, Haufe H, Kemmerling R, Hitzl W, Mikuz G, Moser PL, et al. Increased activated Akt expression in renal cell carcinomas and prognosis. Journal of cellular and molecular medicine. 2009; 13: 2181-8.

50. Melstrom LG, Salabat MR, Ding XZ, Milam BM, Strouch M, Pelling JC, et al. Apigenin inhibits the GLUT-1 glucose transporter and the phosphoinositide 3-kinase/Akt pathway in human pancreatic cancer cells. Pancreas. 2008; 37: 426-31.

51. Wieman HL, Wofford JA, Rathmell JC. Cytokine stimulation promotes glucose uptake via phosphatidylinositol-3 kinase/Akt regulation of Glut1 activity and trafficking. Molecular biology of the cell. 2007; 18: 1437-46.

52. Wu XL, Wang LK, Yang DD, Qu M, Yang YJ, Guo F, et al. Effects of Glut1 gene silencing on proliferation, differentiation, and apoptosis of colorectal cancer cells by targeting the TGF-beta/PI3KAKT-mTOR signaling pathway. Journal of cellular biochemistry. 2018; 119: 2356-67.

\section{Figures}



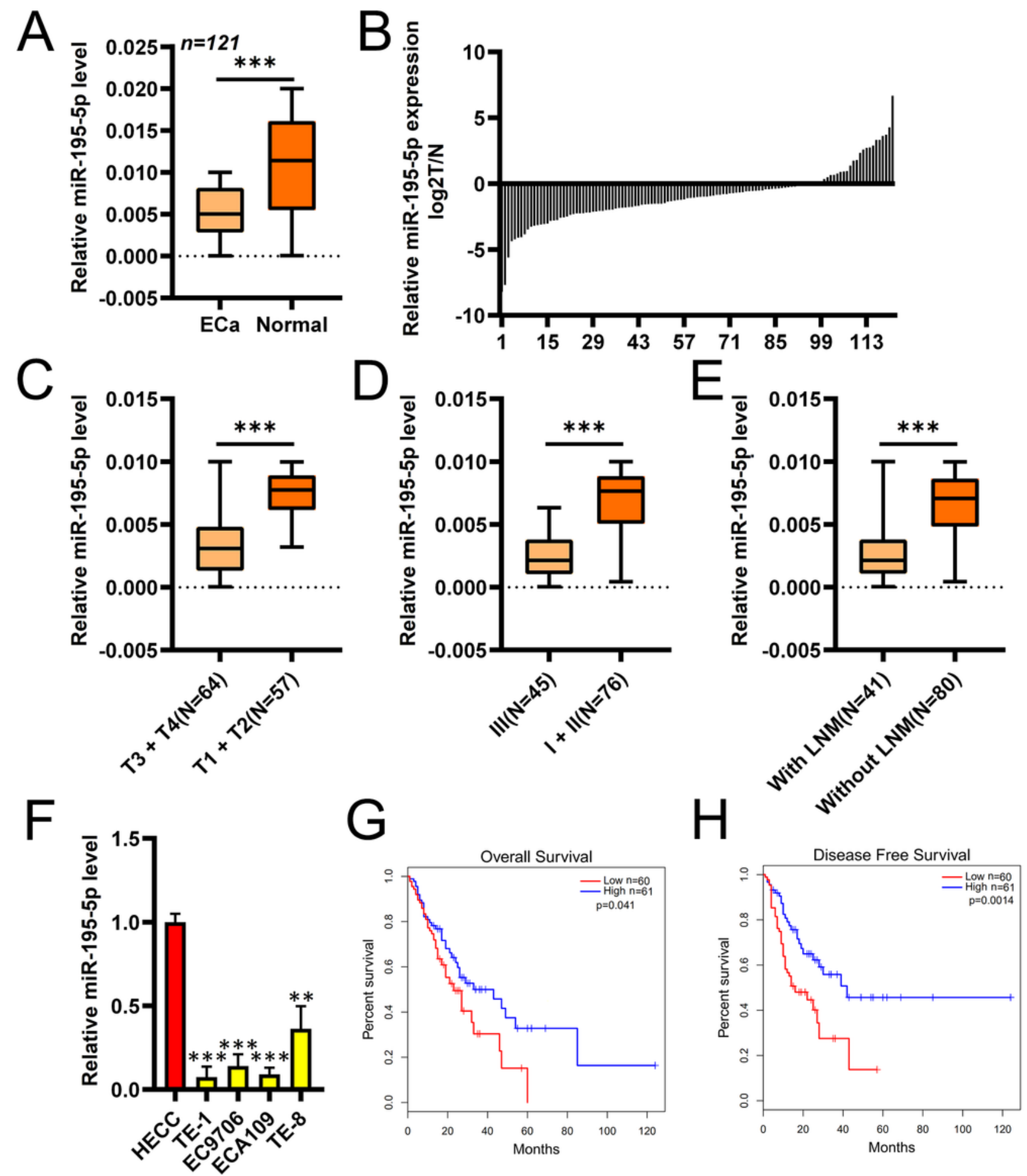

Figure 1

MiR-195-5p is suppressed in esophageal cancer tissues and cells. (A-B) RT-PCR showing miR-195-5p levels in $121 \mathrm{ECa}$ tissues vs. healthy tissue. (C-E) Loss of miR-195-5p correlates with T3 + T4 invasion depth, TNM3 + 4 stage, and metastasis. (F) MiR-195-5p levels are reduced in the indicated ECa cell lines compared to HECC cells. (G-H) Overall survival (OS) and Disease-free survival (DFS) in ECa patients. Kaplan Meier (KM) curves were plotted for survival analysis. ${ }^{*} \mathrm{P}<0.05$. ${ }^{*} \mathrm{P}<0.01 .{ }^{* *} \mathrm{P}<0.001$ 

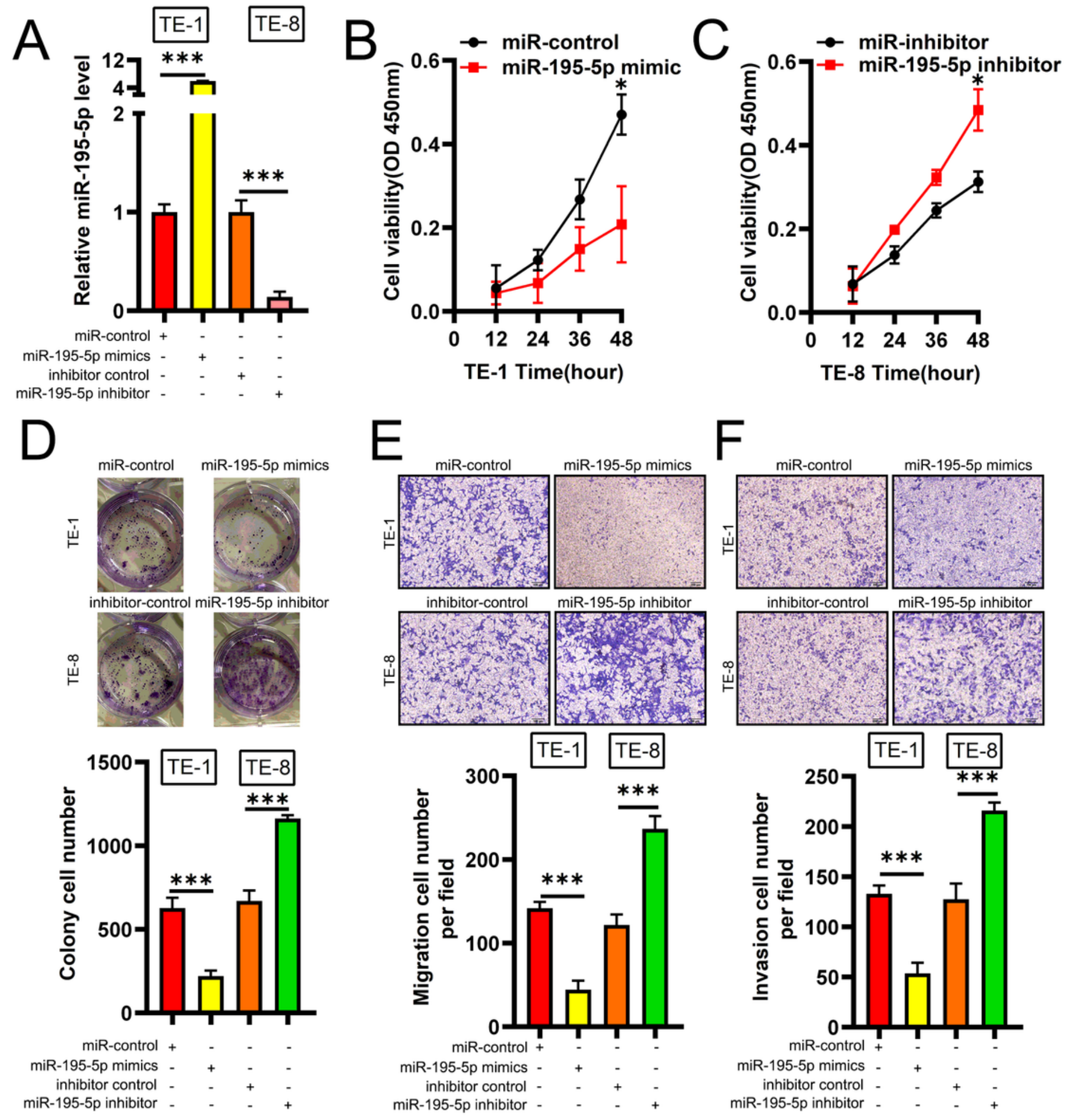

Figure 2

MiR-195-5p inhibits the metastatic phenotypes of ECa cells. (A) miR-195-5p levels in TE-1 and TE-8 cells was performed by RT-PCR. (B-C), CCK-8 assays in miR-195-5p mimic or inhibitor in indicated ECa cells. (D) Clone formation assays in miR-195-5p mimic or inhibitor cells. Upper panel: Representative images of colony formation assays. (E-F) Transwell assays in miR-195-5p mimic or inhibitor in indicated ECa cells. Upper panel: Representative images of Transwell assays. $(n=3)$. P-values as in Fig 1. 
A

Position 1269-1275

IGF1R WT-3' UTR 5' ... UACAGAAAAAAAAAAGCUGCUAU..

hsa-miR-195-5p 3' CGGUUAUAAAGACACGACGAU

IGF1R MUT-3' UTR 5' ...UACAGAAAAAAAAAACGACGAAU.
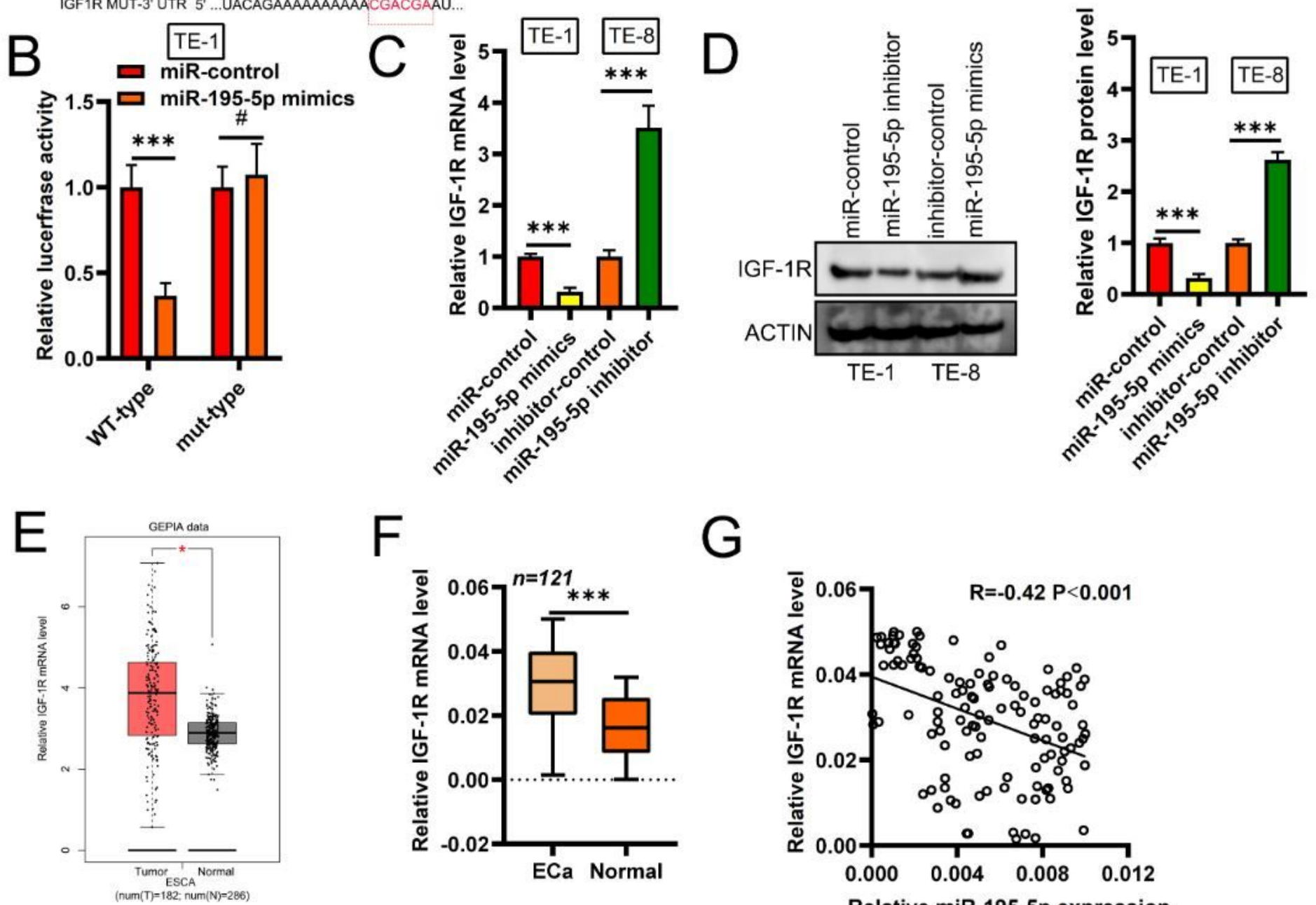

G

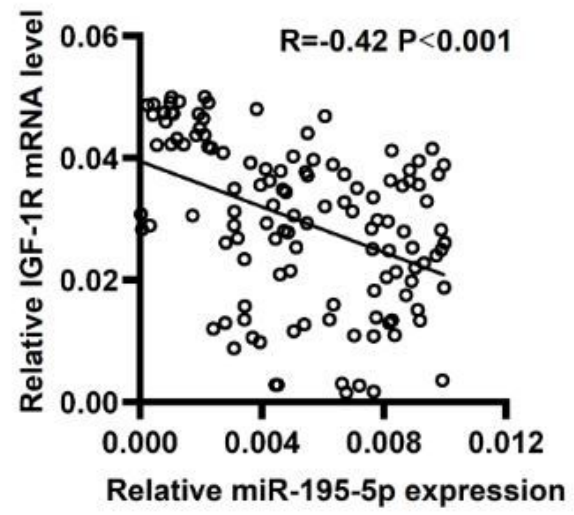

Figure 3

MiR-195-5p suppresses IGF-1R expression in ECa. (A) Bioinformatics predicting miR-195-5p targeting of IGF-1R. (B) Reporter assays showing the relationship between miR-195-5p and IGF-1R in TE-1. (C-D). WB and RT-PCR analysis of IGF-1R in miR-195-5p mimic or inhibitor cells. (E) GEPIA analysis showing higher IGF-1R levels in ECa tissues. (F) RT-PCR showing increased IGF-1R mRNA levels in ECa. (G) Correlation of miR-195-5p and IGF-1R. ( $n=3)$ P-values as in Fig 1. 

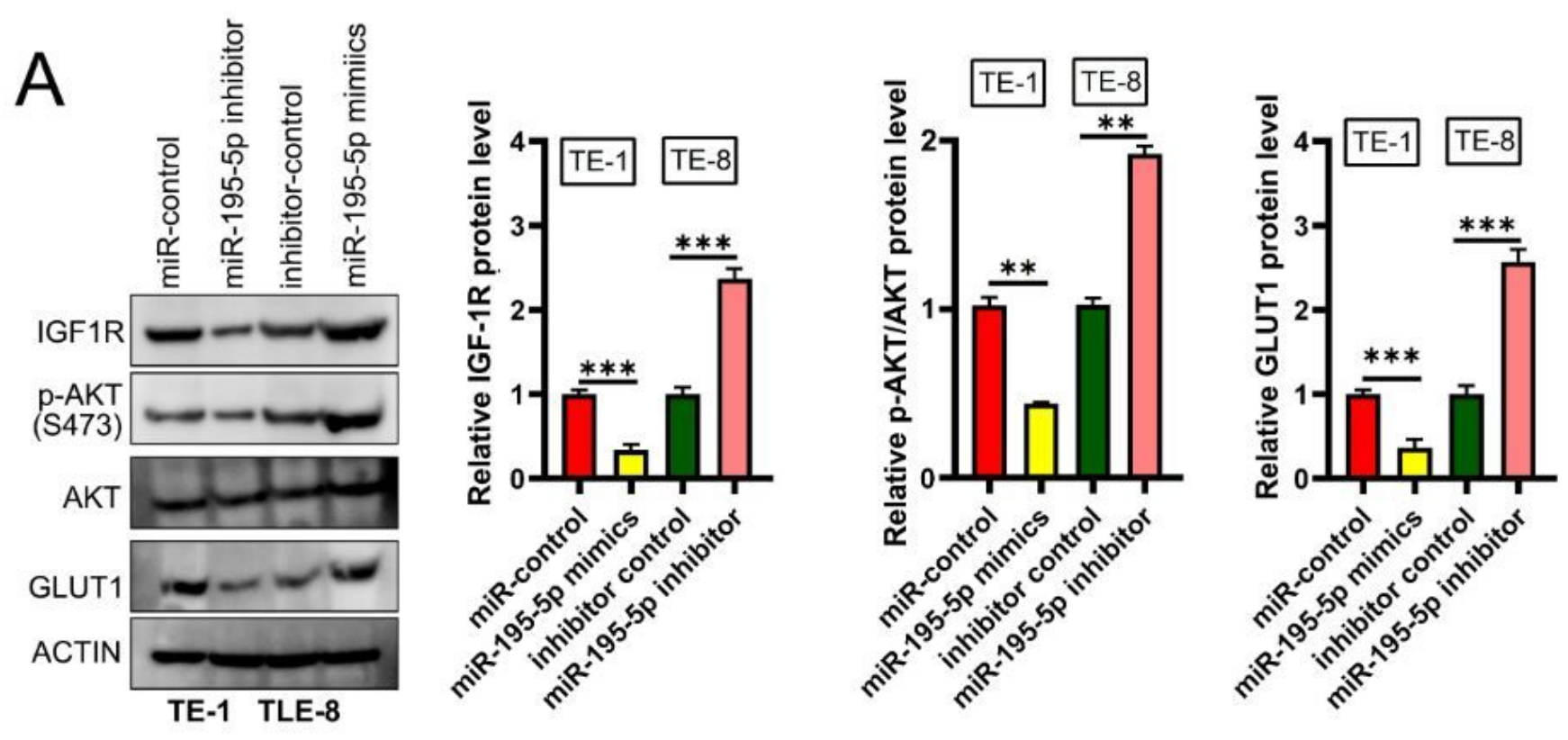

B

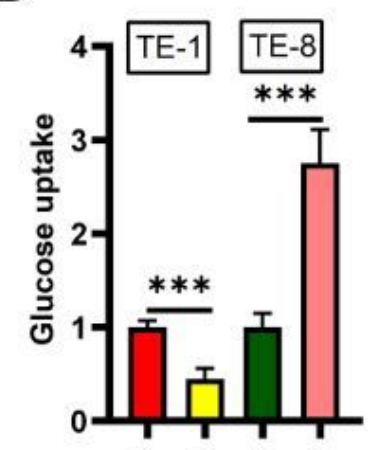

C
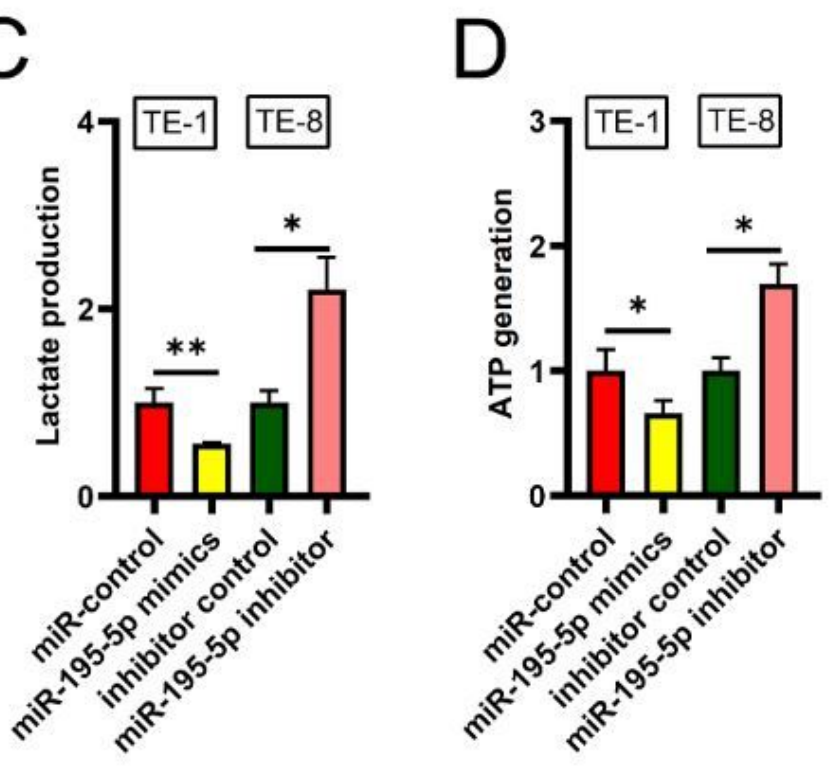

Figure 4

MiR-195-5p controls ECa cell glycolysis via the IGF-1R/AKT/GLUT1 axis. (A) IGF-1R, AKT and GLUT1 expression in mimic or inhibitor in indicated ECa cells. (B-C) Rates of glucose uptake and lactate production in mimic or inhibitor in indicated ECa cells. (D) ATP levels in mimic or inhibitor in indicated ECa cells $(n=3)$. P-values as in Fig.1. 


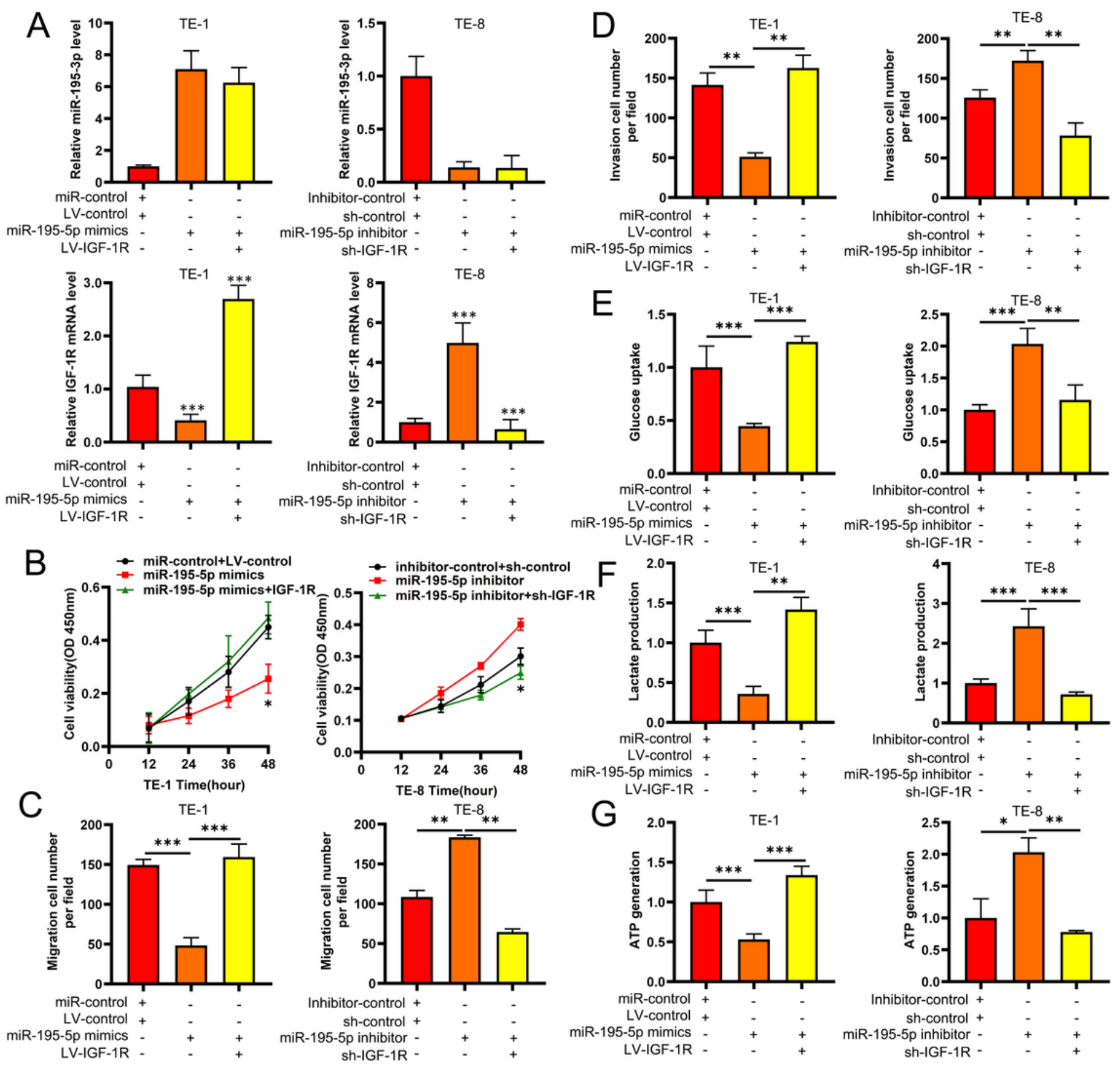

Figure 5

MiR-195-5p suppresses glycolysis via IGF-1R in ECa cells. (A) miR-195-5p and IGF-1R expression via RTPCR. (B) CCK-8 assays in the indicated ECa cell lines. (D-E) Migration and invasion Transwell assays. (F$\mathrm{G})$ Cells expressing inhibitors or mimics were assessed for glucose uptake, the production of lactate and ATP levels. $(n=3)$. P-values as in Fig 1.

\section{Supplementary Files}

This is a list of supplementary files associated with this preprint. Click to download. 
- supplementin.docx

Page 19/19 